\title{
ANTIPODAL COINCIDENCE FOR MAPS OF SPHERES INTO COMPLEXES
}

\author{
MAREK IZYDOREK AND JAN JAWOROWSKI
}

(Communicated by Thomas Goodwillie)

\begin{abstract}
This paper gives a partial answer to the question of whether there exists a Borsuk-Ulam type theorem for maps of $S^{n}$ into lower-dimensional spaces, which are not necessarily manifolds. It is shown that for each $k$ and $n \leq 2 k-1$, there exists a map $f$ of $S^{n}$ into a contractible $k$-dimensional complex $Y$ such that $f x \neq f(-x)$, for all $x \in S^{n}$. In particular, there exists a map of $S^{3}$ into a 2-dimensional complex $Y$ without an antipodal coincidence. This answers a question raised by Conner and Floyd in 1964. The complex $Y$ provides also an example of a countractible $k$-dimensional complex whose deleted product has the Yang-index equal to $2 k-1$.
\end{abstract}

\section{INTRODUCTION AND NOTATION}

We will say that a map $f$ from $S^{n}$ to a space $Y$ has an antipodal coincidence if there exists a point $x \in S^{n}$ such that $f x=f(-x)$. The classical BorsukUlam theorem says that every map $f: S^{n} \rightarrow \mathbf{R}^{k}$ has an antipodal coincidence if $k \leq n$. Conner and Floyd ([1], p. 85) proved that if $k<n$, then every map $f: S^{n} \rightarrow M^{k}$ of $S^{n}$ into a $k$-dimensional differentiable manifold $M^{k}$ has an antipodal coincidence. They also asked a specific question ([1], 89) of whether there is a map of $S^{3}$ into a 2-dimensional complex without an antipodal coincidence. We will construct such a map of $S^{3}$ into a 2-dimensional contractible complex $Y$. The construction is extremely simple and geometric: in our example, $Y$ is a subcomplex of the barycentric subdivision of a standard simplex. We will also show that 3 is the lowest dimension of the sphere for which such an example can be constructed.

If $A$ and $B$ are spaces, we will denote by $A * B$ the join of $A$ and $B$. It consists of segments joining the points of $A$ with the points of $B$, the segments being mutually disjoint except at the endpoints. The join of $A$ with the empty set is $A$ itself. There is a standard (deformation) retraction of $(A * B)-A$ to $B$ along the segments of the join.

If $s$ is a simplex and $t$ is a face of $s$, we will denote by $c(t)$ the face of $s$ which is complementary to $t$; i.e., $s$ is the join of $t$ and $c(t)$; and

Received by the editors June 8, 1993 and, in revised form, September 28, 1993 and October 12, 1993.

1991 Mathematics Subject Classification. Primary 55M20; Secondary 55M35.

Work by the first author was supported, in part, by the Kosciuszko Foundation. 
$\operatorname{dim} c(t)=\operatorname{dim} s-\operatorname{dim} t-1$. The barycenter of a simplex $s$ will be denoted by $b(s)$. By the carrier of a point $x$ in a complex $K$ we mean, as usual, the simplex of $K$ containing $x$ in the interior.

Let $\Delta$ be a standard $(n+1)$-dimensional simplex and let $\Delta^{\prime}$ denote its barycentric subdivision. We can think of $\Delta$ as a subset of $\mathbf{R}^{n+2}$ embedded in the standard way, so that the coordinates in $\mathbf{R}^{n+2}$ are the barycentric coordinates of points of $\Delta$. We will denote by $\alpha: \mathbf{R}^{n+2} \rightarrow \mathbf{R}^{n+2}$ the symmetry of $\mathbf{R}^{n+2}$ about the diagonal of $\mathbf{R}^{n+2}$, i.e., the line where all the coordinates are equal to each other. Then $\alpha$ corresponds to the antipodal map of the $(n+1)$-plane $P$ in which the simplex $\Delta$ lies. Let $o$ be the barycenter of $\Delta$. Two points of $\mathbf{R}^{n+2}$ are said to be antipodal (to each other) if they lie on opposite sides of the center $o$. The simplex $\Delta$ is not invariant under the central symmetry $\alpha$, but the symmetry induces a simplicial map $\beta: \Delta^{\prime} \rightarrow \Delta^{\prime}$ of the barycentric subdivision of $\Delta$. In fact, if $b=b(s)$ is a vertex of $\Delta^{\prime}$ and $b$ is the barycenter of a face $s$ of $\Delta$, then $\beta(b)$ is the barycenter of the face complementary to $b: \beta(b)=b(c(s))$.

The vertices of the barycentric subdivision $\Delta^{\prime}$ are partially ordered, as usual, by their "ranks": the rank of a vertex $b$ of $\Delta^{\prime}$ is the dimension of the face of $\Delta$ of which $b$ is the barycenter. Note the following facts:

Lemma 1. (1) The involution $\beta: \Delta^{\prime} \rightarrow \Delta^{\prime}$ reverses the ranks of the vertices: if $b$ is a vertex of $\Delta^{\prime}$ of rank $q$, then the rank of $\beta(b)$ is $n-q$ (in the case $q=n+1$ it is understood that the center $o$ is the barycenter of both $\Delta$ and the empty simplex).

(2) $\beta: \Delta^{\prime} \rightarrow \Delta^{\prime}$ is simplicially free outside the center $o$ in the sense that for each simplex $s$ of $\Delta^{\prime}$, the intersection of $s$ with $\beta(s)$ consists at most of the center $o$.

(3) If $x$ and $y$ is an antipodal pair in $\Delta$ and if $u$ and $v$ are the carriers of $x$ and $y$ in $\Delta^{\prime}$, respectively, then $v=\beta(u)$.

The construction of our example rests on the fact that a simplex is so much "antisymmetric" with respect to the antipodal map.

Lemma 2. If $s$ is a q-simplex of $\Delta^{\prime}$ which is a part of the q-skeleton $\Delta^{q}$ of $\Delta$, then the intersection $(\beta(s)) \cap \Delta^{q}$ is a face of $\beta(s)$ of dimension $2 q-n$.

Proof. Since $s$ is a simplex of $\Delta^{\prime}$ in $\Delta^{q}$, the ranks of its vertices form a sequence $(0, \ldots, q)$. Thus by Lemma 1 , the ranks of $\beta(s)$ form a sequence $(n-q, \ldots, n)$. The face of $\beta(s)$ which is in $\Delta^{q}$ is made up of the vertices whose ranks are not more than $q$. In the sequence $(n-q, \ldots, n)$ there are exactly $2 q-n+1$ integers not greater than $q$.

Corollary. If $2 q+1 \leq n$, then $\beta\left(\Delta^{q}\right) \cap \Delta^{q}=\varnothing$.

\section{CONSTRUCTION OF THE COMPLEX $Y$}

Throughout Sections 2 and 3, let $q$ be an integer and $k=n-q$.

Definition. Every simplex $s$ of the barycentric subdivision $\Delta^{\prime}$ is the join of a unique pair $u, v$ of simplices of $\Delta^{\prime}$, where $u$ lies in the $q$-skeleton $\Delta^{q}$ of $\Delta$ and $v$ is disjoint from the $q$-skeleton. The simplices $v$ so obtained form a $k$-dimensional subcomplex $Y^{k}$ of $\Delta^{\prime}$. We will call it the $k$-dimensional spine, denoted $Y^{k}$, of $\Delta$ (briefly, the $k$-spine) "dual" to the $q$-skeleton $\Delta^{q}$. We can 
also define the spine inductively by saying that the $k$-spine of a simplex is the union of the cones over the $(k-1)$-spines of the boundary simplices.

Let $v_{0}, \ldots, v_{n+1}$ be the vertices of $\Delta$. Given a set of $q+1$ indices $i_{0}, \ldots, i_{q}$ out of $(0, \ldots, n+1)$, we will denote by $b\left(i_{0}, \ldots, i_{q}\right)$ the center of the face spanned by $v_{i_{0}}, \ldots, v_{i_{q}}$. The $k$-spine $Y^{k}$ is disjoint with the $q$-skeleton of $\Delta$ and intersects each $(q+1)$-face of $\Delta$ spanned by $v_{i_{0}}, \ldots, v_{i_{q}+1}$ in its center $b\left(i_{0}, \ldots, i_{q+1}\right)$. These centers are "the extremities" of the spine $Y^{k}$. There are $\left(\begin{array}{c}n+2 \\ q+2\end{array}\right)$ of them.

Let $C_{k}$ be the convex hull of the $k$-spine $Y^{k}$. Then $C_{k}$ is also the convex hull of the set of its extremities $\left\{b\left(i_{0}, \ldots, i_{q+1}\right)\right\}$. It is a convex $(n+1)$ dimensional polyhedron with vertices $\left\{b\left(i_{0}, \ldots, i_{q+1}\right)\right\}$ contained in $\Delta-\Delta^{q}$. The boundary of $C_{k}$, which we denote by $\Sigma_{k}$, is a simplicial $n$-sphere.

Note that $C_{k}$ is the intersection of the $(n+2)$ half-spaces given by $(q+2) x_{i} \leq$ $1, i=0, \ldots, n+1$, with the simplex $\Delta$. The part of $C_{k}$ lying on an $m$-face of $C_{k}$ is just $C_{k+m-n-1}$.

The special case of $n=3, k=2, q=1$. In this case (which is actually the first interesting one), we can see that $Y^{2}$ is a 2-dimensional complex whose intersection with each 3-dimensional simplex $s$ on the boundary of $\Delta$ consists of four intervals going from the center of $s$ to its 2-dimensional faces. The complex $Y^{2}$ itself is the cone over the graph made up by these four-legged objects. The convex hull $C_{2}$ of $Y^{2}$ is a 4-dimensional polyhedron with 10 vertices. It cannot be a regular polyhedron since there are no regular 4-dimensional polyhedra with 10 vertices. In fact, the boundary $\Sigma_{2}$ of $C_{2}$ has five tetrahedra (one in every 3-simplex of $\Sigma_{2}$ ), five octahedra (one "opposite" each vertex of $\Delta$ ), thirty triangles, thirty edges and ten vertices (the Euler number being zero, as it should be).

\section{THE MAP OF $S^{n}$ INTO $Y^{k}$}

Recall that every simplex $s$ of $\Delta^{\prime}$ is the join of a unique simplex $u$ of the barycentric subdivision of the $q$-skeleton of $\Delta^{q}$ of $\Delta$ with a unique simplex $v$ of $Y^{k}$. Thus there exists a standard deformation retraction of $\Delta-\Delta^{q}$ to $Y^{k}$ along the lines of the join in each simplex of $\Delta^{\prime}$ (with its face in $\Delta^{q}$ deleted). Let $f: \Sigma_{k} \cong S^{n} \rightarrow Y^{k}$ be the restriction of this map to $\Sigma_{k}$. The antipodal symmetry $\alpha$ induces a free involution on $\Sigma_{k} \cong S^{n}$ which will also be called antipodal.

Lemma 3. If $x$ and $y$ is a pair of antipodal points of $\Sigma_{k}$ such that $f x=f y$, then $f x=f y$ must be the center $o$.

Proof. Let $z=f x=f y$. Let $t$ be the carrier of $z$ in $Y^{k}$ and let $u$ and $v$ be the carriers of $x$ and $y$, respectively, in $\Delta^{\prime}$. By the construction of $f$, the simplex $t$ is a face of both $u$ and $v$. Since $x$ and $y$ is an antipodal pair, Lemma 1 implies that the intersection $u \cap v$ is just the center $o$. Thus $z=0$.

Proposition. If $n \leq 2 k-1$, then the map $f: S^{n} \rightarrow Y^{k}$ has no antipodal coincidence: if $x$ and $y$ is an antipodal pair on $S^{n}$, then $f x \neq f y$.

Proof. By Lemma 3, $f$ projects the points $x$ and $y$ to the center $o$ from an antipodal pair of points lying in the $(n-k)$-skeleton $\Delta^{n-k}$ of $\Delta$. However, if 
$n \leq 2 k-1$; then $2(n-k)-1 \leq n$ and by the corollary, $\Delta^{n-k}$ contains no antipodal pair.

\section{CONCLUDING REMARKS}

It is not hard to see that if $n>2 k$, then every map $f: S^{n} \rightarrow Y$ of a finite $k$ dimensional complex $Y$ into $S^{n}$ has an antipodal coincidence. If, in addition, $H_{k}\left(Y ; Z_{2}\right)=0$, then the inequality $n>2 k-1$ is already sufficient. For, if $f: S^{n} \rightarrow Y$ is a map without an antipodal coincidence, then $f$ defines a $Z_{2}$ equivariant map from $S^{n}$ to the deleted product $Y^{*}$ of $Y$. By an argument similar to that used in [2] one can show that $H^{2 k}\left(Y^{*} ; \mathbf{Z}_{2}\right)=0$. Thus the Yang $\mathbf{Z}_{2}$-index, Ind ${ }^{Z_{2}} Y^{*}$, of $Y^{*}$ (see [1] and [3]) is less than $2 k \leq n$ and hence an equivariant map from $S^{n}$ to $Y^{*}$ cannnot exist.

Thus we have the following result.

Theorem. For each $k$ and $n \leq 2 k-1$, there exists a map $f$ of $S^{n}$ into a contractible $k$-dimensional complex $Y$ without an antipodal coincidence. In particular, there exists such a map of $S^{3}$ into a contractible 2-dimensional complex. Moreover, $n=3$ is the lowest integer for which there is a map of $S^{n}$ into a complex of dimension less than $n$ without an antipodal coincidence.

In particular, the deleted produce $Y^{*}$ of the contractible complex $Y$ constructed in Section 2 has the Yang $\mathbf{Z}_{2}$-index equal to $2 k-1$. We do not know whether there exist a $k$-dimensional complex whose deleted product has the Yang $\mathbf{Z}_{2}$-index equal to $2 k$.

\section{REFERENCES}

[1] P. E. Conner and E. E. Floyd, Differentiable periodic maps, Springer-Verlag, Berlin and New York, 1964.

[2] C. W. Patty, A note on the homology of deleted product spaces, Proc. Amer. Math. Soc. 14 (1963), 800.

[3] C. T. Yang, On theorems of Borsuk-Ulam Kakutani-Yamabe-Yujobô and Dyson. I, Ann. of Math. (2) 60 (1954), 262-282.

Wydzial Fizyki Technicznej i Matematyki Stosowanej, Politechnika Gdańska, Ul. Majakowskiego 11-12, 80-952 Gdansk, Poland

E-mail address: izydorek@iu-math.math.indiana.edu

Department of Mathematics, Indiana University, Bloomington, Indiana 47405-5701

Current address: Department of Mathematics, The University of Auckland, Auckland, New Zealand

E-mail address: jaworows@ucs.indiana.edu 